\title{
A conductive heat transfer model for particle flows over immersed surfaces
}

\author{
A. B. Morris ${ }^{1}$, S. Pannala ${ }^{2}$, Z. $\mathrm{Ma}^{3}$, and C. M. Hrenya ${ }^{1 \dagger}$ \\ 1 University of Colorado at Boulder, Dept. of Chemical and Biological Engineering, Boulder, CO, USA \\ 2 Oakridge National Laboratory, Knoxville, TN, USA \\ 3 Department of Energy National Renewable Energy Laboratory, Golden, CO, USA
}

\begin{abstract}
A fundamental continuum model for conductive heat transfer between an immersed boundary and flowing particles is developed. The model is derived for systems where conduction through the interstitial gas between nearby surfaces is the dominant heat transfer mechanism. Conductive heat transfer depends on the thermal properties of the solids and gas phases, particle size and morphology, and packing structure. The new model incorporates both particle size and arrangement effects using first principles, and is applicable to flows spanning dilute to dense regimes. Specifically, a novel particle-wall distribution function is employed to capture the effects of particle arrangement over a range of solids concentrations. Discrete element method (DEM) simulations are used to close the model in terms of continuum variables and to generate constitutive relations for the Nusselt number and local heat transfer coefficient. The resulting expression is implemented into a continuum gas-solid model and tested against DEM data for particle flow down a ramp, flow around a hexagon, and crossflow around a cylinder. The model accurately predicts the local heat transfer coefficient over a range of flow parameters, and is valid for the full range of solids concentrations.
\end{abstract}

Keywords: Heat conduction; Granular flow; Discrete element method

${ }^{\dagger}$ Corresponding author: hrenya@colorado.edu

Prof. Christine Hrenya

University of Colorado at Boulder

Jennie Smoly Caruthers Biotechnology Building

UCB 596

Boulder, CO 80309

\section{Variables}

$C_{p} \quad=$ specific heat $[\mathrm{J} / \mathrm{kg}-\mathrm{K}]$

$d \quad=$ separation distance $[\mathrm{m}]$

$D_{p} \quad=$ particle diameter $[\mu \mathrm{m}]$

$e \quad=$ normal coefficient of restitution

g = gravitational acceleration $\left[\mathrm{m} / \mathrm{s}^{2}\right]$

$H \quad=$ thermal conductance $[\mathrm{W} / \mathrm{K}]$

$h=$ heat transfer coefficient $\left[\mathrm{W} / \mathrm{m}^{2}-\mathrm{K}\right]$

$H_{g} \quad=$ interphase thermal conductance $[\mathrm{W} / \mathrm{K}]$

\section{Nomenclature}

$$
\begin{array}{ll}
\beta & =\text { gas-solids drag coefficient } \\
\chi & =\text { particle-wall distribution } \\
& \text { function } \\
\delta & =\text { particle-wall overlap [m] } \\
\varepsilon & =\text { volume fraction } \\
\phi_{g} & =\text { gas-solids granular energy } \\
& \quad \text { dissipation term } \\
\phi_{k} & =\text { fractional contact area } \\
\gamma & =\text { dissipation term due to inelastic } \\
\kappa & \quad \text { collisions } \\
\kappa & =\text { thermal conductivity [W/m-K] }
\end{array}
$$




\begin{tabular}{|c|c|c|c|}
\hline$k_{n}$ & $=$ collision spring constant $[\mathrm{N} / \mathrm{m}]$ & $\Theta$ & $=$ granular temperature $\left[\mathrm{m}^{2} / \mathrm{s}^{2}\right]$ \\
\hline$l$ & $\begin{array}{l}=\text { gap distance between the particle } \\
\text { surface and the wall }[\mathrm{m}]\end{array}$ & $\theta$ & $=$ ramp angle \\
\hline$m_{p}$ & $=$ particle mass $[\mathrm{kg}]$ & $\rho$ & $=$ density $\left[\mathrm{kg} / \mathrm{m}^{3}\right]$ \\
\hline$n_{p}$ & $=$ areal number density of particles $\left[\mathrm{m}^{-2}\right]$ & $\tau$ & $=$ shear stress tensor \\
\hline$P$ & $=$ pressure & $\mu$ & $=$ Dufour coefficient \\
\hline $\mathbf{q}$ & $=$ heat flux (thermal or granular) $\left[\mathrm{W} / \mathrm{m}^{2}\right]$ & $\mu_{g}$ & $=$ gas viscosity $[\mathrm{Pa}-\mathrm{s}]$ \\
\hline$Q$ & $=$ heat transfer rate $[\mathrm{W}]$ & \multicolumn{2}{|c|}{ Subscripts } \\
\hline$R$ & $=\operatorname{radius}[\mathrm{m}]$ & $c$ & $=$ contact \\
\hline$r$ & $=$ radial distance $[\mathrm{m}]$ & $g$ & $=$ gas phase \\
\hline$s$ & $=$ minimum conduction distance $[\mathrm{m}]$ & lens & $=$ fluid lens \\
\hline$t$ & $=$ time $[\mathrm{s}]$ & $p$ & $=$ particle \\
\hline$T$ & $=$ thermodynamic temperature $[\mathrm{K}]$ & $p p$ & $=$ particle-particle \\
\hline & $=$ velocity $[\mathrm{m} / \mathrm{s}]$ & $p f p$ & $=$ particle-fluid-particle \\
\hline \multicolumn{2}{|c|}{ Dimensionless groups } & $p w$ & $=$ particle-wall \\
\hline $\mathrm{Bi}$ & $=$ Biot number, $h D_{p} / \kappa_{p}$ & $p f w$ & $=$ particle-fluid-wall \\
\hline $\mathrm{Nu}$ & $=$ Nusselt number, $h D_{p} / \kappa_{g}$ & ref & $=$ reference value \\
\hline $\mathrm{Nu}_{\mathrm{g}}$ & $=$ Nusselt number for interphase transfer & $s$ & $=$ solids phase \\
\hline $\operatorname{Pr}$ & $=$ Prandtl number & $w$ & $=$ wall \\
\hline $\operatorname{Re}$ & $=$ Reynolds number & $\Theta$ & $=$ granular temperature \\
\hline
\end{tabular}

\section{Introduction}

Solid particle flows coupled with heat transfer are commonplace in numerous industrial operations. Modern examples include rotary-kiln or fluidized-bed units used for the drying of wet particles (pharmaceuticals, food grains, chemicals, ores, biomass, etc.) and reactors that utilize solids as a catalyst or as a reactant (gasification of biomass and/or coal, fluid catalytic cracking, etc.). Moreover, the use of solid particles as a heat transfer "fluid" can offer advantages over conventional fluids. For example, molten salts are currently used for heat transfer from the receivers of concentrating solar power (CSP) plants, though such salts become unstable at $\sim 500-600^{\circ} \mathrm{C}$ [1]. Inexpensive solids like sand, however, are inert at much higher temperatures in which radiation effects can be exploited $[2,3]$.

For particle flows over immersed surfaces like heat exchanger tubes, the heat transfer at the boundary plays a critical role. Mechanisms for heat transfer at the boundary include conduction, convection, and radiation. The focus of the current work is on conduction, which plays the key role for relatively dense particle flows at the boundaries. Such conduction can be further divided into two contributions: particle-wall conduction and particle-fluid-wall conduction. The former refers to direct conduction across the particle-wall contact area, whereas the latter refers to conductive heat transfer through the thin gas layer between nearby solid surfaces. We limit our scope here to particle-fluid-wall conduction, which is the dominant mechanism of conduction in a variety of systems. Particle-fluid-wall conduction is significant in systems where the 
non-dimensional parameter $R_{p} \kappa_{g} / R_{c} \kappa_{p}$ is greater than unity, where $\kappa_{g}$ and $\kappa_{p}$ are the thermal conductivity of the gas and particle, respectively, $R_{p}$ is the radius of the particle, and $R_{c}$ is the radius of the contact area. In particular, particle-fluid-wall conduction is important for materials such as sand, where the particle thermal conductivity is much less than other materials such as alumina pellets.

Despite the practical importance of conductive heat transfer in solids flows, a predictive understanding remains elusive. Particles can be modeled as discrete entities or as a continuum. The discrete element method (DEM) is a well-established tool for simulating solids flows by solving Newton's equations of motion for individual particles [4-6] as well as energy balances for each particle [7-10]. In a discrete particle approach, particles are modelled as thermal reservoirs and heat is transferred via thermal pipes when they contact. The thermal pipes connecting particles are characterized via a thermal contact resistance and depends on particle size, shape, and properties of the interstitial gas. In [8], the thermal resistance between particle-particle contacts is an input model parameter and is calibrated to match the bed thermal conductivity from continuum penetration theory. In $[9,10]$, the thermal pipe resistance is solved for every contact by considering conduction across the mutual contact area and through the stagnant interstitial gas between particles. Although DEM is an accurate and fundamental approach, it is computationally expensive, and for many practical applications a continuum approach must be used instead. Continuum models, however, require closures for the effective thermal conductivity of the solids phase [11-13] and appropriate boundary conditions, both of which must account for the discrete nature of particle contacts in an averaged (continuum) manner. Such closures are challenging to determine because, unlike a traditional molecular fluid, the heat transfer is tightly coupled to the packing arrangement of the particles. For example, the solids conductivity may be different near a boundary than in the bulk flow because the wall affects the packing structure [14]. An accurate heat-transfer boundary condition is essential for the systems considered here and is the focus of the current effort.

Continuum models, also referred to as penetration models, estimate the heat transfer by including a contact resistance associated with conduction from the wall to the first layer of particles near the wall and a penetration resistance associated with conduction through the solids phase $[15,16]$. For short contact durations, the heat transfer is limited by the contact resistance and depends on the particle diameter, morphology, gas conductivity, and surface coverage. Expressions for the contact resistance can be found in [12,16], but the surface coverage factor is not valid over a range of solids concentration. For longer contact times, the heat transfer is limited by the rate at which energy penetrates through the medium. For dynamic systems, contact resistance is expected to limit the heat transfer because convective motion and mixing of particles reduces the penetration resistance.

Prior work on heat-transfer boundary conditions for continuum models has taken two forms. The first is based on the concept of an effective thermal conductivity as alluded to above. In this approach, the solids flux at the wall is assumed to follow Fourier's law: 


$$
q^{\prime \prime}=\left.\varepsilon_{s} \kappa_{s} \frac{\partial T}{\partial x_{i}}\right|_{w}
$$

where $\varepsilon_{s}$ is the solids volume fraction (i.e., solids concentration), $T$ is the thermodynamic temperature, and $\kappa_{s}$ is the effective thermal solids conductivity [11-17]. This effective solids conductivity $\kappa_{s}$, which is derived based on an analogy with the kinetic theory of dense gases, depends on the solids concentration as well as gas and solid conductivities, but not on particle size. This lack of dependency on particle size is contrary to experimental data [16-18], which indicate that smaller particles lead to increased heat transfer.

The other type of boundary condition utilized in continuum models is based on Nusselt number $(\mathrm{Nu})$ correlations [21, 22]. Early Nusselt-number correlations are based on experimental data for flow along a ramp [21]. Such correlations are based on a packed-bed limit, and thus do not depend on solids concentration. Moreover, spatial averaging leads to an overall, rather than local, heat flux prediction. Molerus [22] derived a Nusselt-number correlation for closely-packed particles in contact with a surface by analytically computing the particle-fluid-wall conduction [22]. This approach is advantageous because it does not contain empirical parameters and can be used to predict the local heat transfer coefficient, but the derivation is also based on an assumption of closely-packed particle beds - i.e., it is not a function of solids concentration. For many dynamic systems, the particles are not closely packed and the Nusselt number correlation of Molerus [22] can over-predict the heat transfer, as our results below illustrate.

In the current effort, we build upon previous works on heat transfer boundary conditions for use in continuum models. Specifically, we derive a physically-based boundary condition which takes the form of a Nusselt number correlation. Unlike past works, the new boundary condition, which contains no tunable parameters, is a function of solids concentration and particle size, as well as surface roughness. The solids concentration dependency is incorporated via a new "particle-wall" distribution function, which is analogous to the particle-particle radial distribution function employed in the kinetic theory of dense gases. Specifically, this particle-wall distribution function provides the distribution of separation distances between the wall and nearby particles for a given solids concentration; this distribution is critical for the accurate prediction of particle-fluid-wall conduction. The new Nusselt number model is applied to a number of systems and is validated via a comparison with DEM data.

\section{Methods}

The simulations are performed with Multiphase Flow with Interphase Exchange (MFIX) [23,24], a state-of-the-art computational fluid dynamics (CFD) open-source code for multiphase flows developed at the National Energy Technology Laboratory (NETL). MFIX solves the solids phase in either a Lagrangian framework using the discrete element method (DEM) or an Eulerian framework via a two-fluid continuum model (TFM) based on the kinetic-theory analogy [24-26]. The discrete element method is 
computationally expensive because the position and velocity of each particle are tracked simultaneously via a force balance for each particle, and thus its use is limited to relatively small systems. The two-fluid-model, or continuum model, is more computationally efficient but requires closures for constitutive relations such as the heat flux and shear stress tensor.

The continuum model solves conservation equations for mass, momentum, granular energy, and thermal energy. These governing equations are summarized in Table 1 . The granular energy is the kinetic energy associated with the random fluctuating components of the particle velocity; it is analogous to temperature in a molecular gas in that it is a measure of fluctuation velocity but dissimilar in that it is not a measure of "hotness". This granular energy is characterized by the granular temperature, $\Theta$; it is generated by velocity gradients, conducted along granular temperature gradients, and dissipated by inelastic collisions and viscous losses to the gas. Constitutive relations based on the wellestablished kinetic-theory analogy [27] are used to close terms for the granular shear stress $\tau_{s}$, granular energy heat flux $q_{\Theta}$, and dissipation rate due to inelastic collisions $\gamma_{\Theta}$. Plastic or frictional stress models available in MFIX are not used for densely packed regions and kinetic-theory closures for stress are used for all solids concentrations. The maximum packing is specified in the continuum model, and a value of $\varepsilon_{s, \max }=0.58$ is used unless otherwise noted. Viscous dissipation of granular energy due to interactions with the background gas, $\phi_{g}$, is solved for as suggested in [28].

The thermal energy equation balances the solids phase internal energy, namely the energy associated with the thermodynamic temperature $T_{s}$. For a non-reacting, monodisperse system, the thermal energy balance is shown in Table 1, where $\varepsilon_{s}$ is the solids concentration, $\rho_{s}$ is the solids material density, $C_{p, s}$ is the specific heat of the solid, $T_{\mathrm{s}}$ is the internal thermal temperature, $T_{g}$ is the temperature of the gas phase, $\mathbf{v}_{\mathrm{s}}$ is the bulk solids velocity, $\mathbf{q}_{\mathrm{s}}$ is the solids phase conductive flux, and $H_{g}$ is the interphase thermal conductance. The aim of this work is to develop a constitutive relation for the solids phase heat flux at particle-wall boundaries, $\left.\mathbf{q}_{\mathrm{s}}\right|_{\text {wall }}$, and then validate the model by comparison to DEM simulation data.

Table 1. Summary of equations

\begin{tabular}{|l|l|}
\hline \multicolumn{2}{|l|}{ Two-Fluid Model: Governing Equations } \\
\hline $\begin{array}{l}\text { Continuity: } \\
\text { Solid phase }\end{array}$ & $\frac{\partial}{\partial t}\left(\varepsilon_{s} \rho_{s}\right)+\vec{v}_{s} \cdot \nabla\left(\varepsilon_{s} \rho_{s}\right)=0$ \\
\hline $\begin{array}{l}\text { Gas phase } \\
\text { Momentum: } \\
\text { Solid phase }\end{array}$ & $\frac{\partial}{\partial t}\left(\varepsilon_{g} \rho_{g}\right)+\vec{v}_{g} \cdot \nabla\left(\varepsilon_{g} \rho_{g}\right)=0$ \\
\hline Gas phase & $\frac{\partial}{\partial t}\left(\varepsilon_{s} \rho_{s} \vec{v}_{s}\right)+\nabla \cdot\left(\varepsilon_{s} \rho_{s} \vec{v}_{s} \vec{v}_{s}\right)=-\varepsilon_{s} \nabla P_{g}+\nabla \cdot \overrightarrow{\bar{\tau}}_{s}+\beta_{g, s}\left(\vec{v}_{s}-\vec{v}_{g}\right)+\varepsilon_{s} \rho_{s} \vec{g}$ \\
\hline
\end{tabular}




\begin{tabular}{|c|c|}
\hline Granular Energy & $\frac{3}{2}\left[\frac{\partial}{\partial t}\left(\varepsilon_{s} \rho_{s} \Theta\right)+\nabla \cdot\left(\varepsilon_{s} \rho_{s} \Theta \vec{v}_{s}\right)\right]=\left(\overline{\bar{\tau}}_{s}+P_{s} \overline{\bar{I}}\right): \nabla \vec{v}_{s}-\nabla \cdot \vec{q}_{\Theta}-\gamma_{\Theta}+\phi_{g}$ \\
\hline $\begin{array}{l}\text { Thermal Energy: } \\
\text { Solid phase }\end{array}$ & $\frac{\partial}{\partial t}\left(\varepsilon_{s} \rho_{s} C_{p, s} T_{s}\right)+\vec{v}_{s} \cdot \nabla\left(\varepsilon_{s} \rho_{s} C_{p, s} T_{s}\right)=-\nabla \cdot \vec{q}_{s}+H_{g}\left(T_{g}-T_{s}\right)$ \\
\hline Gas phase & $\frac{\partial}{\partial t}\left(\varepsilon_{g} \rho_{g} C_{p, g} T_{g}\right)+\vec{v}_{g} \cdot \nabla\left(\varepsilon_{g} \rho_{g} C_{p, g} T_{g}\right)=-\nabla \cdot \vec{q}_{g}-H_{g}\left(T_{g}-T_{s}\right)$ \\
\hline \multicolumn{2}{|c|}{ Constitutive Relations } \\
\hline $\begin{array}{l}\text { Interphase heat } \\
\text { transfer [29] }\end{array}$ & $\begin{aligned} H_{g}= & \frac{6 \kappa_{g} \varepsilon_{s} N u_{g s}}{D_{p}^{2}} \\
N u_{g s}= & \left(7-10 \varepsilon_{g}+5 \varepsilon_{g}^{2}\right)\left(1+0.7 \operatorname{Re}^{0.2} \operatorname{Pr}^{1 / 3}\right)+ \\
& \left(1.33-2.4 \varepsilon_{g}+1.2 \varepsilon_{g}^{2}\right) \operatorname{Re}^{0.7} \operatorname{Pr}^{1 / 3}\end{aligned}$ \\
\hline Gas Conduction & $\begin{array}{l}\vec{q}_{g}=-\varepsilon_{g} \kappa_{g} \nabla T_{g} \\
\kappa_{g}=\kappa_{g, r e f} \sqrt{T_{g} / T_{r e f}}\end{array}$ \\
\hline $\begin{array}{l}\text { Solids Conduction } \\
{[12,13,15]}\end{array}$ & $\begin{array}{l}\vec{q}_{s}=-\varepsilon_{s} \kappa_{s} \nabla T_{s} \\
\frac{\kappa_{s}}{\kappa_{g}}=\frac{\phi_{k}\left(\kappa_{p} / \kappa_{g}\right)+\left(1-\phi_{k}\right) \lambda_{r}}{\sqrt{\varepsilon_{s}}} \\
\lambda_{r}=-\frac{2}{1-b \frac{\kappa_{g}}{\kappa_{p}}}\left[\frac{\left(\frac{\kappa_{p}}{\kappa_{g}}-1\right) b \frac{\kappa_{g}}{\kappa_{p}}}{\left(1-b \frac{\kappa_{g}}{\kappa_{p}}\right)^{2}} \ln \left(b \frac{\kappa_{g}}{\kappa_{p}}\right)+\frac{b-1}{1-b \frac{\kappa_{g}}{\kappa_{p}}}+\frac{b+1}{2}\right] \\
b=1.25\left(\frac{1-\varepsilon_{g}}{\varepsilon_{g}}\right)^{10 / 9} ; \phi_{k}=7.26 \times 10^{-3}\end{array}$ \\
\hline $\begin{array}{l}\text { Granular energy } \\
\text { heat flux [27] }\end{array}$ & $\begin{array}{l}\vec{q}_{\Theta}=-\kappa_{\Theta} \nabla \Theta-\mu \nabla n \\
\text { Transport coefficients from }\end{array}$ \\
\hline Solids stress [27] & Kinetic-theory closure \\
\hline $\begin{array}{l}\text { Interphase drag } \\
\text { model [28] }\end{array}$ & Gas-solids drag model \\
\hline \multicolumn{2}{|c|}{ DEM: Governing Equations } \\
\hline Momentum & $m_{p, i} \frac{d \vec{v}_{p, i}}{d t}=\sum_{j} \vec{F}_{c, i j}+\vec{I}_{g, s}-m_{p, i} g$ \\
\hline Thermal Energy & $m_{i} C_{p, i} \frac{d T_{i}}{d t}=\sum_{j}\left(\dot{Q}_{i j}^{p p}+\dot{Q}_{i j}^{p f p}\right)+H_{g}\left(T_{g}-T_{i}\right)+\dot{Q}_{p w}+\dot{Q}_{p f w}$ \\
\hline \multicolumn{2}{|l|}{ DEM: Closures } \\
\hline Contact Model & $F_{c, i j}=-k_{n} \delta-\eta_{n} \frac{d \delta}{d t}$ \\
\hline
\end{tabular}




\begin{tabular}{|l|l|}
\hline $\begin{array}{l}\text { Particle-particle } \\
\text { conduction [30] }\end{array}$ & $\dot{Q}_{p p}^{i j}=H\left(T_{p}^{j}-T_{p}^{i}\right)=2 \kappa_{p} R_{c}\left(T_{p}^{j}-T_{p}^{i}\right)$ \\
\hline $\begin{array}{l}\text { Particle-fluid- } \\
\text { particle conduction } \\
\text { [31] }\end{array}$ & $\dot{Q}_{p f p}^{i j}=H_{p f p}\left(T_{p}^{j}-T_{p}^{i}\right)=\left(T_{p}^{j}-T_{p}^{i}\right) \int_{r_{\text {in }}}^{r_{\text {out }}} \frac{2 \pi \kappa_{g}}{\max \left(s, l^{i j}\right)} d r$ \\
\hline $\begin{array}{l}\text { Particle-wall } \\
\text { conduction }\end{array}$ & $\dot{Q}_{p w}=H_{p w}\left(R_{p}-\delta-\sqrt{R_{p}^{2}-r^{2}}\right]=\frac{4 \kappa_{p} \kappa_{w}}{\kappa_{p}+\kappa_{w}} R_{c}\left(T_{w}-T_{p}\right)$ \\
\hline $\begin{array}{l}\text { Particle-fluid-wall } \\
\text { conduction }\end{array}$ & $\dot{Q}_{p f w}=H_{p f w}\left(T_{w}-T_{p}\right)=\left(T_{w}-T_{p}\right) \int_{r_{\text {in }}}^{r_{\text {out }}} \frac{2 \pi \kappa_{g} r}{\max (l, s)} d r$ \\
& $l=R_{p}-\delta-\sqrt{R_{p}^{2}-r^{2}}$ \\
\hline
\end{tabular}

Unlike the continuum model shown above, DEM simulations track individual particle trajectories and temperatures. Contacts are modeled using a linear spring dashpot (LSD) model where the repulsive force is proportional to the overlap between contacting particles and a dashpot is used to dissipate energy. The thermal energy balance is solved for every particle and is shown in Table 1. In the DEM thermal energy equation, the subscripts $i$ and $j$ denote individual particles, $m$ is the particle mass, $Q_{\mathrm{pp}}$ is conductive heat transfer that occurs across the contact area between touching particles and $Q_{\mathrm{pfp}}$ is the conduction through the stagnant gas between close particles. The boundary heat transfer terms for particle-wall and particle-fluid-wall conduction are $Q_{p w}$ and $Q_{p f w}$. These terms will be discussed in more detail in the following section. Since DEM simulations resolve individual particle motion and heat transfer, and thus do not require closure for continuum fluxes $\left(\tau_{s}\right.$ and $\mathbf{q}_{\Theta}$ ), we treat DEM simulation as ideal data for validating the continuum heat transfer model.

The simulations and models discussed in this work are for a monodisperse granular media, and friction is neglected in both the DEM and continuum simulations. Radiative heat transfer is neglected, but interphase coupling between the solids and gas phases are explicitly solved for using existing correlations [29]. The simulations are fully three-dimensional because accurate prediction of the particle arrangement is necessary for computing $\mathbf{q}_{\mathrm{s}}$.

\section{Theory}

This section outlines the theory behind the development of the new conduction model for particle-wall heat transfer, which will ultimately be implemented as a boundary condition for the continuum model outlined above. The mechanisms and existing models for heat transfer from the wall to a single particle, as used in DEM, are first discussed. These expressions are then used as a basis for generalization to a continuum of particles. Closures to these expressions are then derived from DEM data and used to complete the new continuum particle-wall conduction model. 


\subsection{Particle-wall heat transfer: Particle level (DEM)}

Heat transfer mechanisms in solid particle flows include conduction within the solids phase, bulk convection of particles, interphase coupling with the gas phase, and radiative heat transfer. Radiation is generally neglected in DEM simulations for temperatures below approximately $600^{\circ} \mathrm{C}$ because the contributions from radiative heat transfer are relatively small [32]. Similarly, for moderately dense to densely-packed particle flows, conduction dominates and interphase heat exchange can be neglected. Although this work targets such dense systems where solids phase conduction is the dominant heat transfer mechanism, the new model is valid for dilute and dense-phase flows.

Conduction can occur via two mechanisms: (i) direct conduction through the mutual contact area between two touching bodies and (ii) indirect conduction through the thin wedge of interstitial gas between two bodies. For sand particles, the ratio $R_{p} \kappa_{g} / R_{c} \kappa_{p}$ is greater than unity and direct conduction across the mutual contact area tends to be negligible compared to indirect conduction. Further, if the particles are moving, thermal contact is not sustained and thus less heat transfer occurs via direct contacts. For indirect heat conduction, also referred to as particle-fluid-particle or particle-fluid-wall conduction, heat is transferred through the wedge of interstitial gas between nearby bodies. This process is shown schematically in Figure 1.

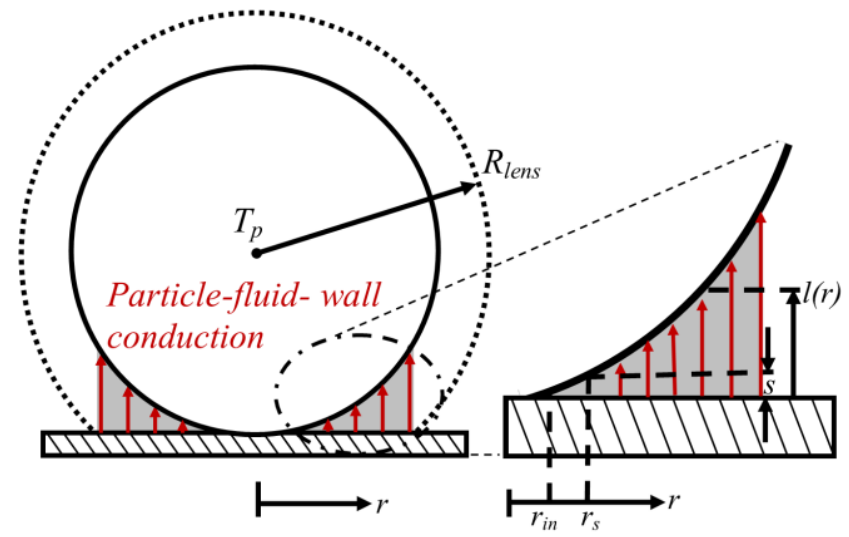

Figure 1. A schematic of a particle-fluid-wall conduction

Several models exist for particle-fluid-particle or particle-fluid-wall conduction $[31,33,34]$ and MFIX uses the Rong and Horio (1999) model. This model views each particle as being surrounded by a thin fluid lens through which heat conduction occurs. The conductive heat transfer is assumed to occur when the fluid lens of one body intersects with the solid surface of another. The resulting heat transfer is further assumed to be one-dimensional in the direction normal to the contact area. In addition, thermal energy within the particles is assumed to be rapidly distributed such that the particles are isothermal. This assumption is valid for low Biot number, $\mathrm{Bi} \equiv h D_{p} / \kappa_{p}$, where $h$ is a heat transfer coefficient, $D_{p}$ is the particle diameter, and $\kappa_{p}$ is the material thermal conductivity of a particle. This assumption will be revisited in more detail after 
discussing the particle-fluid-wall model. Accordingly, the heat transfer rate from the wall to a particle via particle-fluid-wall conduction is

$$
\dot{Q}_{p f w}=H_{p f w}\left(T_{w}-T_{p}\right)=\left(T_{w}-T_{p}\right) \int_{r_{\text {in }}}^{r_{\text {out }}} \frac{2 \pi \kappa_{g} r}{\max (l, s)} d r,
$$

where $T_{w}$ and $T_{p}$ are the wall and particle temperatures, respectively, $r$ is a radial distance shown in Figure 1, $l$ is a function of $r$ and is the normal distance from the wall to particle surface, and $s$ is an assumed minimum conduction distance. The lower bound of the integral, $r_{i n}$, is the radial distance associated with the contact area $\left(r_{i n}=0\right.$ for particles that are close to, but not touching, the surface), and $r_{\text {out }}$ is the distance where the particle lens intersects the wall. The upper bound of the integral is constrained to be less than the particle radius. The minimum conduction distance, $s$, is invoked to prevent the integral from becoming singular where the particle is in contact with the wall. Physically, $s$ represents the height of roughness asperities and also accounts for rarefaction effects that reduce the conduction through the gas [12].

This model for particle-fluid-wall conduction requires two input parameters, the lens thickness $R_{\text {lens }}$ as well and the minimum conduction distance $s$. The lens thickness has an upper bound such that $r_{\text {out }}$ does not exceed the particle radius. This constraint prevents the gap distance $l$ from becoming undefined. Studies of surface-to-bed heat transfer in a vertical heater suggest a lens thickness that is $20 \%$ of the particle radius [35]. Other similar models for particle-fluid-particle conduction assume the lens thickness is large enough such that the upper bound of the integral in Eq. 2 is the particle radius [36]; from geometric arguments, the resulting lens thickness is approximately $41 \%$ of the particle radius. Increasing the lens thickness from $20 \%$ of the particle radius to $41 \%$ does not result in a dramatic increase in the overall heat transfer because most of the heat transfer occurs where the gap distance is small. The lens thickness in this work is typically $40 \%$ of the particle radius and our results are not very sensitive to this value. The minimum conduction distance $s$, however, has a significant impact on the heat transfer. Figure 2 a shows how the non-dimensional thermal conductance, shown later in Eq. 6, varies with the assumed minimum conduction distance. The thermal conductance is defined as the ratio of the heat transfer rate to the temperature difference, or $H=\dot{Q} / \Delta T$. It was suggested by [12] that the minimum gap distance depends on the thermal accommodation coefficient and mean free path of the background gas. The thermal accommodation coefficient is the probability that a gas molecule reflects off a surface with an energy in thermal equilibrium with the surface temperature. At small distances where the gap distance is comparable to the mean free path of gaseous molecules, the gas thermal conductivity decreases because of rarefaction effects. Alternatively, the minimum gap distance has also been argued to be dependent on the surface morphology, and specifically has been assumed equal to the height of a surface asperity. For the simulations here, the particles are assumed to be smooth, and thus $s$ is comparable to the mean free path of gas molecules and a value of $2.75 \times 10^{-8} \mathrm{~m}$ is used. 


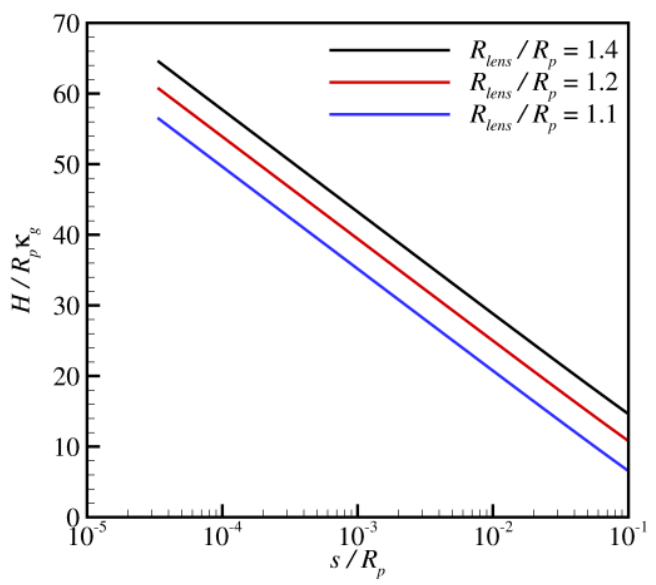

(a)

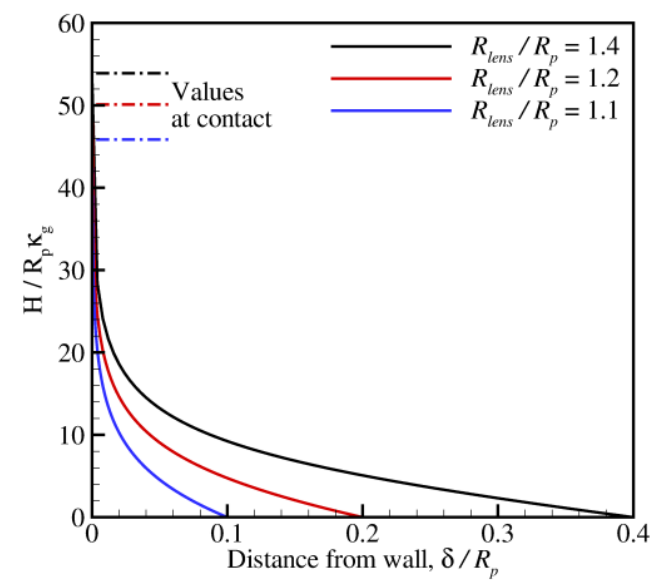

(b)

Figure 2. Sensitivity of (a) the minimum conduction distance, $s$, and (b) the separation distance between particles and the wall. Different lines indicate different values for the assumed fluid lens radius.

The heat transfer also closely depends on the proximity of the particle relative to the wall. Figure $2 \mathrm{~b}$ shows how the thermal conductance depends on the separation distance in non-dimensional terms. The different (solid) lines correspond to different values for the lens radius and the horizontal (dashed-dotted) lines denote maximum values of the non-dimensional thermal conductance evaluated when a particle contacts the wall. The scaled minimum conduction distance, $s / R_{p}$, is $1.83 \times 10^{-4}$. The horizontal dashed-dotted lines are shown for visual clarity and denote values of the non-dimensional thermal conductance evaluated when a particle contacts the wall. The thermal conductance decreases rapidly as the particle slightly moves away from the wall. For example, the thermal conductance decreases by approximately $80 \%$ from values at contact when the particle is only $2 \%$ of the particle radius from the wall. For flowing particles, this difference is significant because particles will constantly be moving into and out of contact with the wall. This observation is a critical component of our new continuum model because it illustrates the impact of particle proximity to the wall on the accuracy of heat transfer predictions.

The thermal conductance is non-dimensionalized by the product between the gas thermal conductivity and the particle radius, and all distances are non-dimensionalized by the particle radius. The non-dimensional variables are denoted by hats and the corresponding equation for the non-dimensional thermal conductance is

$$
\begin{aligned}
& \hat{H}=\frac{H}{\kappa_{g} R_{p}}=\int_{\hat{r}_{\text {in }}}^{\hat{r}_{\text {out }}} \frac{2 \pi \hat{r}}{\max (\hat{l}, \hat{s})} d \hat{r} \\
& \hat{l}=\frac{l}{R_{p}}=1-\hat{\delta}-\sqrt{1-\hat{r}^{2}}
\end{aligned}
$$


The normal distance separating the wall from a location on the particle surface, $l$, depends on the particle overlap, $\delta$. Positive values of overlap indicate that the particle is in contact with the surface, while negative values refer to the distance separating the particle and wall. Analytic solutions for $\hat{H}$ are obtained by integrating Eq. 3 when a particle contacts the surface $(\delta \geq 0)$, and when a particle is close to but not in contact with a surface $(\delta<0)$ :

$$
\hat{H}=\left\{\begin{array}{cc}
\frac{\pi}{\hat{s}}\left[(1-\hat{\delta})^{2}-A\right]+2 \pi\left[B-A+(1-\delta) \ln \left(\frac{1-\hat{\delta}-B}{\hat{s}}\right)\right] & , \hat{\delta} \geq 0 \\
2 \pi\left[\frac{\hat{r}_{s}^{2}}{2 \hat{s}}+B-C+(1-\hat{\delta}) \ln \left(\frac{1-\hat{\delta}-B}{1-\hat{\delta}-C}\right)\right] & , \hat{\delta}<0
\end{array},\right.
$$

where

$$
\begin{aligned}
& A=1-\hat{\delta}-\hat{s} \\
& B=\sqrt{1-\hat{r}_{\text {out }}^{2}} \\
& C=\sqrt{1-\hat{r}_{s}^{2}}
\end{aligned}
$$

The variable $r_{s}$ corresponds to the radial position such that $l\left(r_{s}\right)=s$. When nondimensionalized, these distances are

$$
\begin{aligned}
& \hat{r}_{\text {out }}=\left\{\begin{array}{cc}
\sqrt{\hat{R}_{\text {lens }}^{2}-(1-\hat{\delta})^{2}} & , \hat{\delta}<1-\sqrt{\hat{R}_{\text {lens }}^{2}-1} \\
1 & , \hat{\delta} \geq 1-\sqrt{\hat{R}_{\text {lens }}^{2}-1}
\end{array}\right. \\
& \hat{r}_{s}=\left\{\begin{array}{cc}
\sqrt{1-A^{2}} & , \hat{\delta}>-\hat{s} \\
0 & , \delta \leq-\hat{s}
\end{array}\right.
\end{aligned}
$$

The above expressions for $\hat{H}$ can be used to compute the instantaneous heat transfer rate from the wall to a single particle. To develop the continuum model, the heat transfer to the solids phase is computed by including the heat transfer to all particles adjacent to the surface (i.e., either in contact or within the lens thickness of the wall).

\subsection{Particle-wall heat transfer: continuum}

To incorporate contributions from all particles into the solids phase heat flux, the positions of all particles relative to the wall are required. The particle-wall distribution function $\chi$ is defined as the probability that a particle center exists within a distance $y$ to $y+d y$ from the wall. This distribution function is analogous to the radial distribution function which is commonly used in the kinetic-theory-based models to correlate contacts between particles [37]. A schematic showing the particle-wall distribution function is shown in Figure 3. 


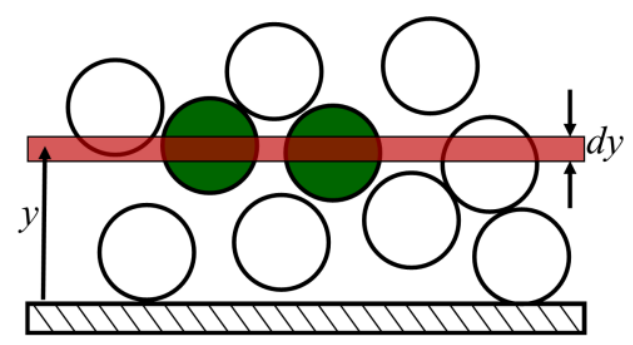

Figure 3. Schematic of particle-wall distribution function with two green particles whose centers lie within a distance $y$ to $y+d y$ from the wall.

The boundary heat flux is obtained by coupling the single particle conductance with the particle-wall distribution function $\chi$, and integrating from those particles in contact with the surface, $\delta=0$, to a distance where the fluid lens surrounding the particle no longer intersects the wall, $\delta=1-R_{\text {lens }}$.

$$
q_{p f w}^{\prime \prime}=\frac{\dot{Q}}{A}=h_{p f w}\left(T_{w}-T_{s}\right)=n_{p} R_{p} \kappa_{g}\left(T_{w}-T_{s}\right) \frac{\int_{0}^{1-\hat{R}_{\text {ens }}} \chi(\hat{\delta}) \hat{H}(\hat{\delta}) d \hat{\delta}}{\int_{0}^{1-\hat{R}_{\text {eens }}} \chi(\hat{\delta}) d \hat{\delta}} .
$$

The variable $n_{p}$ is the number of particles that are within the particle lens thickness of the wall per unit (wall) area, $A$. The particle-fluid-wall local heat transfer coefficient is defined as $h_{p f w}$. The integrand in the numerator is the contribution to the heat flux from particles that are within a distance $\delta$ to $\delta+d \delta$ from the wall. The integral in the denominator is used to normalize the particle-wall distribution function so that the cumulative probability is unity. Note that the particle temperature, $T_{p}$, has been replaced with the continuum solids phase temperature, $T_{s}$, and assumed to be constant over the range of integration.

For Eq. 7 to be useful, the particle-wall distribution function $\chi$ needs to be determined. As a first test on the viability of this approach using the particle-wall distribution function, we obtain $\chi$ from 3-D DEM simulations of particles flowing down an inclined ramp, as shown schematically in Figure 4. 


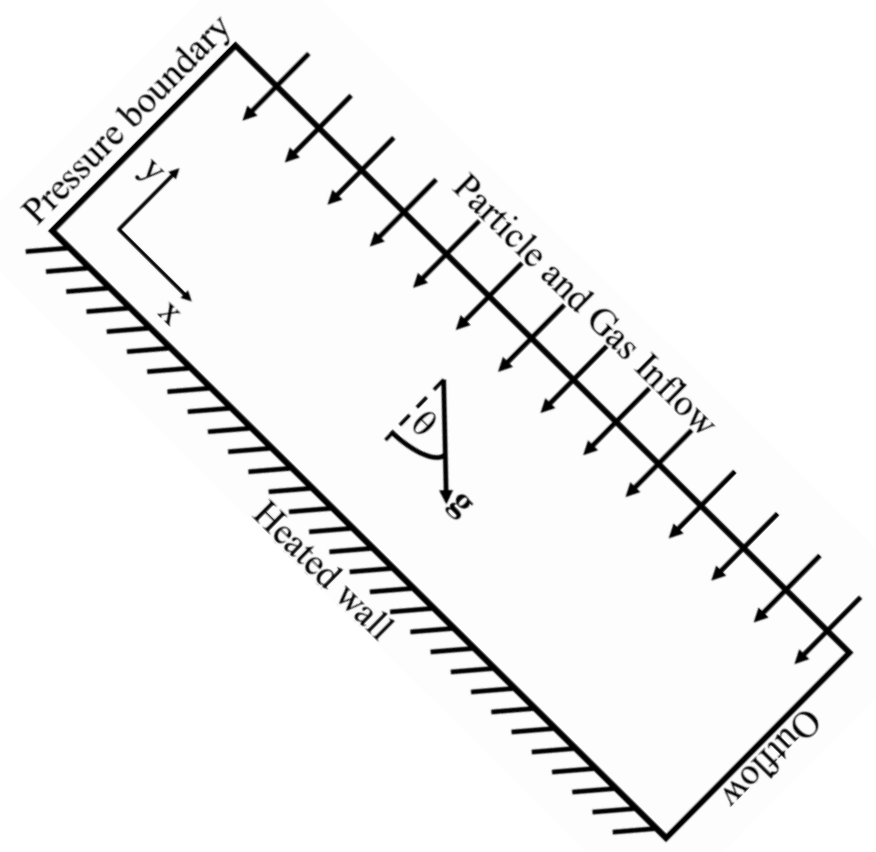

Figure 4. Schematic showing the computational domain for particle flow down an inclined ramp.

In this system, particles uniformly enter the domain from a plane parallel to the ramp. The inlet mass flux of particles was $51.6 \mathrm{~kg} / \mathrm{m}^{2}$-s. The ramp was inclined at an angle of $50^{\circ}$, the particle diameter was $300 \mu \mathrm{m}$, and the particle-particle and particle-wall coefficients of restitution were 0.9 . The coefficient of restitution is defined as the ratio of post- to pre-collision relative velocities and models inelastic particle contacts. The solids concentration is dilute near the top of the ramp and increases as a densely packed chute flow develops. The particle-wall distribution function is obtained via 50 discretized bins normal to the wall, and is calculated at different $x$ locations along the ramp. Particle-wall distribution functions are plotted in Figure 5 at three various $x$ locations where the solids concentration is closely packed, moderately dense, and relatively dilute. The lens thickness is $40 \%$ of the particle radius and the particle-wall distribution function is truncated for particles outside of the lens thickness. Once a steady-state flow develops, time averaging was used to extract smooth particle-wall distribution function profiles at different locations along the surface of the ramp. Near the bottom of the ramp where the solids concentration is densely packed $\left(\varepsilon_{s}=0.58\right)$, the particle-wall distribution function shows that most of the particles within the lens radius of the wall are very close to or in contact with the wall. The solids concentration is less dense near the top of the ramp, and the corresponding particle-wall distribution functions show that the probability of a particle residing a certain distance from the wall is more uniform because volume exclusion effects are less of a factor. 


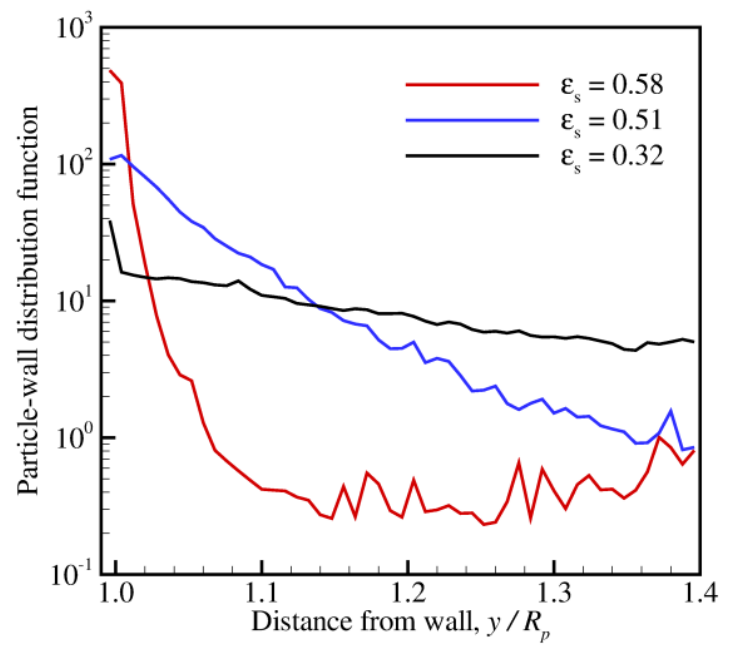

Figure 5. The particle-wall distribution function measured at various solids concentrations for particle flow down an inclined ramp.

In addition to measuring the particle wall distribution, the heat flux was also calculated from Eq. 7 at various locations along the ramp. This prediction was compared to the extracted (DEM) heat fluxes for particles flowing down a ramp inclined at $50^{\circ}$, as displayed in Figure 6. The new model closely matches DEM data in both dilute regions $(x<4 \mathrm{~cm})$ and when the particle flow reaches its packed limit $(\mathrm{x}>4 \mathrm{~cm})$. The heat flux asymptotes to a maximum value when the solids reach the packed limit. Better agreement is expected if the particle-wall distribution function is better resolved. This comparison is encouraging and shows that the physics resolved in the new formulation, and particularly the introduction of the particle-wall distribution function, is a valid and accurate approach. 


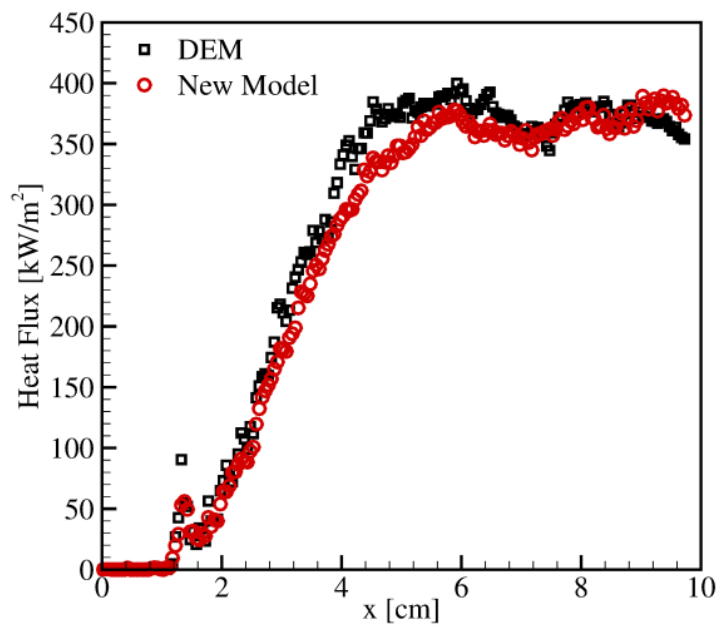

Figure 6. A comparison of the heat flux predicted by DEM and a model, Eq. 7, that uses the particle-wall distribution function measured from DEM data.

In order for the new heat transfer model (Eq. 7) to be useful in a continuum framework, the new particle-wall distribution function must be expressed in terms of continuum variables rather than be obtained from DEM of the system of interest. As detailed below, two approaches have been attempted here to close the heat transfer model in terms of known continuum variables. The first approach, a two-point approximation to the distribution function, was successful when simulations were performed in two dimensions but ultimately failed when applied to three-dimensional systems. Regardless, this first attempt is discussed below because it highlights the physical phenomena that significantly impact the heat transfer and serves as documentation for future work. The second approach hypothesizes that the particle-wall distribution function is only a function of the solids concentration. This assumption allows the Nusselt number to be cast in terms of a solids concentration. Many DEM simulations with different parameters show that the resulting Nusselt number expression collapses versus solids concentration and a constitutive relation is approximated by fitting DEM data. This approach was ultimately successful and, for brevity, is the only model shown in the results section.

\subsubsection{Two-point approximation}

The first attempt to close the model simplifies Eq. 7 by reducing the radial distribution to a two-point approximation, namely separating into two groups particles in contact with the wall and those close to, but not in physical contact with, the wall. This formulation introduces a new variable for the fraction of particles in contact with the surface. Dimensional analysis was used to determine an expression for the fraction of particles in contact with the wall, and it was found that the fraction of particles in contact is most sensitive to the non-dimensional group $\Pi_{1}=\Theta / D_{p} g$. The $\Pi_{1}$ group is the ratio of the granular temperature, $\Theta$, to the product of gravitational acceleration and particle diameter. The granular temperature characterizes the kinetic energy associated fluctuation velocities about the bulk velocity. For very small values of granular 
temperatures all particles move at a similar bulk velocity while higher granular temperatures occur for more agitated particle flows. The $\Pi_{1}$ group can thus be interpreted as the ratio of the fluctuation energy pushing particles away from contact to the gravitational energy drawing particles towards the wall. As the granular temperature, and thus $\Pi_{1}$, increase, the vibrational energy associated with fluctuation velocities pushes particles away from the wall and results in a smaller fraction of particles in contact. This trend has also been observed experimentally in [38], where it was found that vibrating heated surfaces increased the granular temperature and subsequently reduced the heat flux. Though this approach worked well in two dimensions, the two-point approximation was ultimately not pursued because there were significant errors when the heat flux was compared to DEM data in three dimensions. The errors arose because of challenges generating a universal function that collapses the fraction of particles in contact with the surface. In addition, reducing the particle-wall distribution to two points significantly reduces the accuracy of the model. Recall from Figure $2 b$ that the heat transfer is very sensitive to particle separation distance and accurate prediction of the particle arrangement is critical to accurately model the heat transfer.

\subsubsection{Nusselt number approach}

The particle-fluid-wall heat flux in Eq. 7 is first rearranged in terms of a Nusselt number, as shown in

$$
\mathrm{Nu}=\frac{h_{p f w} D_{p}}{\kappa_{g}}=\frac{1}{2} D_{p}^{2} n_{p} \frac{\int_{0}^{1-\hat{R}_{\text {lens }}} \chi(\hat{\delta}) \hat{H}(\hat{\delta}) d \hat{\delta}}{\int_{0}^{1-\hat{R}_{\text {lens }}} \chi(\hat{\delta}) d \hat{\delta}} .
$$

To overcome the aforementioned challenges with the two-point approximation of the particle-wall distribution function, the functional dependence of the particle-wall distribution function is assumed to only depend on solids concentration. This hypothesis is motivated by noting that the analogous radial distribution function used in the kinetictheory-based model for the solids phase is only a function of solids concentration for monodisperse granular material. Further, the number of particles adjacent to the surface, $n_{p}$, is approximated as done in [39] and is

$$
n_{p}=\frac{1}{D_{p}^{2}}\left(\frac{\varepsilon_{s}}{\varepsilon_{s, \max }}\right)^{\frac{2}{3}}
$$

where $\mathcal{E}_{s, \max }$ is the solids concentration at maximum packing. This expression is based on geometric considerations and enables one to define a Nusselt number, as shown in Eq. 8, that is primarily a function of solids concentration (as well as other input parameters such as the minimum conduction distance and lens radius). The minimum conduction distance and lens radius are implicitly embedded in the particle-wall conductance terms $\hat{H}$. Although the expression for $n_{p}$ is approximated by Eq. 9, similar collapse of the Nusselt number can be obtained for any expression for $n_{p}$ that follows a similar function form of $n_{p}=f\left(\varepsilon_{s}\right) / D_{p}^{2}$. 


$$
\mathrm{Nu}=\frac{1}{2}\left(\frac{\varepsilon_{s}}{\varepsilon_{s, \max }}\right)^{2 / 3} \frac{\int_{0}^{1-\hat{R}_{\text {lens }}} \chi(\hat{\delta}) \hat{H}(\hat{\delta}) d \hat{\delta}}{\int_{0}^{1-\hat{R}_{\text {lens }}} \chi(\hat{\delta}) d \hat{\delta}} .
$$

The maximum value of the Nusselt number is shown in Eq. 11 and corresponds to maximum packing and a particle-wall distribution function with all particles in contact (i.e. $\chi(0)=1)$.

$$
\mathrm{Nu}_{\max }=\frac{1}{2} \hat{H}(0) .
$$

As previously noted, the variable $\hat{H}$ depends on the assumed lens thickness and minimum conduction distance. To reduce these dependencies from Eq. 10, the Nusselt number is normalized by the maximum value of the Nusselt number evaluated using the specified minimum conduction distance and fluid lens radius. When cast in this form, the normalized Nusselt number is primarily a function of solids concentration and is shown in Figure 7 for a set of DEM simulations of particle flow along an inclined plane.

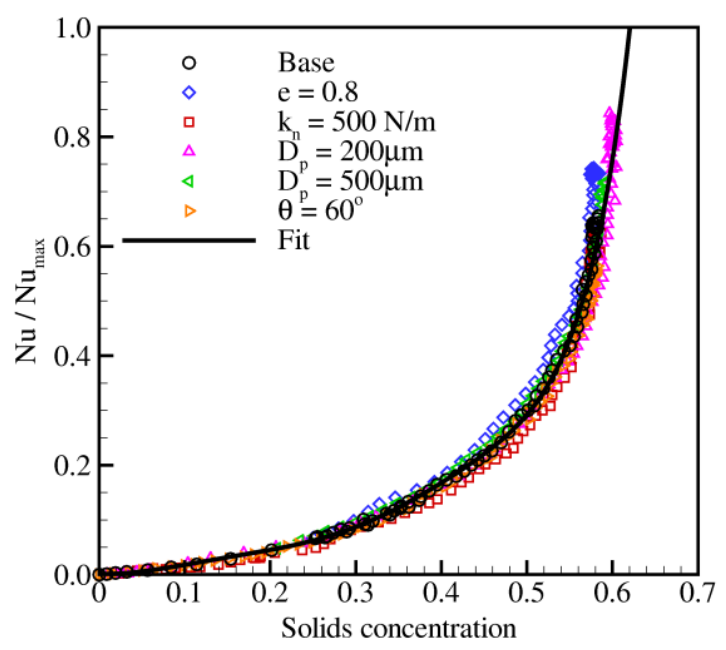

Figure 7. Scaling relationship between the Nusselt number, normalized by the theoretical maximum, and solids concentration. Symbols are 3-D DEM simulations with various flow and model parameters.

The normalized Nusselt numbers collapse very well over the complete range of solids concentrations and flow parameters. The base case simulation in Figure 7 is characterized by $300 \mu \mathrm{m}$ particles flowing down a ramp inclined $45^{\circ}$. The LSD spring constant and coefficient of restitution were $k_{n}=100 \mathrm{~N} / \mathrm{m}$ and $e=0.9$, respectively, for the base case. Although not all cases are shown, the data collapse well for particle diameters ranging from 200 to $500 \mu \mathrm{m}$, inclination angles varying from $30^{\circ}$ to $60^{\circ}$, LSD spring constants from 100 to $500 \mathrm{~N} / \mathrm{m}$, and for simulations with different mass inflow. The restitution was parametrically varied from 0.7 to 0.95 . The resulting normalized Nusselt correlation is found to be closely approximated by the following seventh-order polynomial. 


$$
\begin{aligned}
\frac{\mathrm{Nu}}{\mathrm{Nu}_{\max }}=c_{0} & +c_{1} \varepsilon_{s}+c_{2} \varepsilon_{s}^{2}+c_{3} \varepsilon_{s}^{3}+c_{4} \varepsilon_{s}^{4}+c_{5} \varepsilon_{s}^{5}+c_{6} \varepsilon_{s}^{6}+c_{7} \varepsilon_{s}^{7} \\
c_{o} & =1.0838 \times 10^{-3} \quad c_{1}=-2.1709 \times 10^{-2} \\
c_{2} & =2.4268 \times 10^{0} \quad c_{3}=1.9101 \times 10^{0} \\
c_{4} & =-1.2243 \times 10^{2} \quad c_{5}=6.1504 \times 10^{2} \\
& c_{6}=-1.1706 \times 10^{3} \quad c_{7}=7.9093 \times 10^{2}
\end{aligned}
$$

The key assumptions for this expression are (i) the particle-wall distribution function is only a function of solids concentration and (ii) the areal density of particles near the surface follows the functional form $n_{p}=f\left(e_{s}\right) / D_{p}^{2}$. In addition, the Biot number is assumed to be small, so that particles can be modeled as isothermal. The Biot number can be defined in terms of the Nusselt number and is

$$
\mathrm{Bi}=\frac{h D_{p}}{\kappa_{p}}=\mathrm{Nu} \frac{\kappa_{g}}{\kappa_{p}},
$$

where $\kappa_{p}$ is the thermal conductivity of the solid material. The Nusselt number is largest for particles with a large fluid lens radius and small minimum conduction distance. For $\mathrm{SiO}_{2}$ (sand) particles, the maximum Biot number for simulations in this work is 0.4.

\section{Results}

To test the accuracy and robustness of the new continuum model, DEM simulations are performed for three systems: uniform flow impinging on an inclined ramp, a dense particle stream impinging on the apex of a hexagon, and particles in crossflow over a cylinder. The thermodynamic properties of the gas are summarized in Table 2. The base case material properties for the solids phase are summarized in Table 3 and the numerical and flow parameters are summarized in Table 4. These parameters are used in the following simulations unless otherwise specified.

Table 2. Thermodynamic properties of gas

\begin{tabular}{|l|l|}
\hline \hline Gas species & Air \\
\hline \hline$\kappa_{g}$ & $\kappa_{g}=\kappa_{g, r e f} \sqrt{T_{g} / T_{g, r e f}}, \quad \kappa_{g, r e f}=0.0252 \mathrm{~W} / \mathrm{m}-\mathrm{K}, \quad T_{g, r e f}=300 \mathrm{~K}$ \\
\hline$C_{p, g}$ & $1004.2 \mathrm{~J} / \mathrm{kg}-\mathrm{K}$ \\
\hline Molar mass & $28.97 \mathrm{~kg} / \mathrm{kmol}$ \\
\hline Gas viscosity & $\mu_{g}=\mu_{g, r e f}\left(\frac{T_{g}}{T_{g, r e f}}\right)^{3 / 2} \frac{T_{g, r e f}+S}{T+S}$ \\
& $S=110.4 \mathrm{~K}, \quad \mu_{g, r e f}=1.72 \times 10^{-5} \mathrm{~Pa}-\mathrm{s}, \quad T_{g, r e f}=300 \mathrm{~K}$ \\
\hline
\end{tabular}


Table 3. Base case properties for solids phase

\begin{tabular}{|l|l|l|l|}
\hline \hline Species & $\mathrm{SiO}_{2}(\mathrm{Sand})$ & Young's Modulus & $70 \mathrm{GPa}$ \\
\hline \hline$\kappa_{p}$ & $1.402 \mathrm{~W} / \mathrm{m}-\mathrm{K}$ & Poisson Ratio & 0.17 \\
\hline$C_{p, s}$ & $794.96 \mathrm{~J} / \mathrm{kg}-\mathrm{K}$ & $e$ & 0.9 \\
\hline$D_{p}$ & $300 \mu \mathrm{m}$ & $e$ & \\
\hline
\end{tabular}

Table 4. Base case numerical and flow parameters

\begin{tabular}{|l|l|l|l|}
\hline \hline \multicolumn{4}{|l|}{ Numerical Parameters } \\
\hline \hline Cell size & $0.06 \mathrm{~cm} \times 0.06 \mathrm{~cm} \times 0.06 \mathrm{~cm}$ & \\
\hline Number of cells & $294 \times 390 \times 25$ & \\
\hline$k_{n}$ & $100 \mathrm{~N} / \mathrm{m}$ & \\
\hline \multicolumn{4}{|l|}{} \\
\hline \hline Flow Parameters & $300 \mathrm{~K}$ & $P_{g, \text { outlet }}$ & \\
\hline \hline$T_{g, \text { inlet }}$ & $\varepsilon_{s, \text { inlet }}$ & $10 \%$ \\
\hline$T_{s, \text { inlet }}$ & $300 \mathrm{~K}$ & $e_{\text {wall }}$ & 0.9 \\
\hline$T_{\text {wall }}$ & $600 \mathrm{~K}$ &
\end{tabular}

\subsection{Flow along an inclined ramp}

A schematic of the computational domain for flow along an inclined ramp is shown in Figure 4. This system is designed to test the performance of the model for different flow parameters, such as particle size, restitution coefficient, inclination angle, and numerical parameters such as the LSD spring constant. The continuum model is solved using two different sets of inputs. First, to eliminate the effects of hydrodynamic variability between continuum and DEM simulations, continuum variables such as granular temperature, thermodynamic temperature, and concentration (hydrodynamic variables) are extracted from DEM data and used as inputs for the new model for heat transfer at the boundary. Second, the continuum-only model is implemented such that the hydrodynamic and thermal variables are solved simultaneously in the continuum framework.

\subsubsection{Continuum model: DEM inputs for hydrodynamic variables}

The local heat transfer coefficients are computed using the new model, Eq. 12, a previous Nusselt number correlation for packed beds [22], and an effective solids phase thermal conductivity model $[12,13]$. The results are compared to DEM 'ideal' data in Figure 8 , where the heat transfer coefficient is computed by dividing the boundary heat flux by the temperature difference between the wall and first layer of particles near the wall. In this simulation, particles impinge on the ramp at a distance near $x=1.5 \mathrm{~cm}$. As gravity pulls particles down the ramp, the solids concentration and heat transfer increase. 


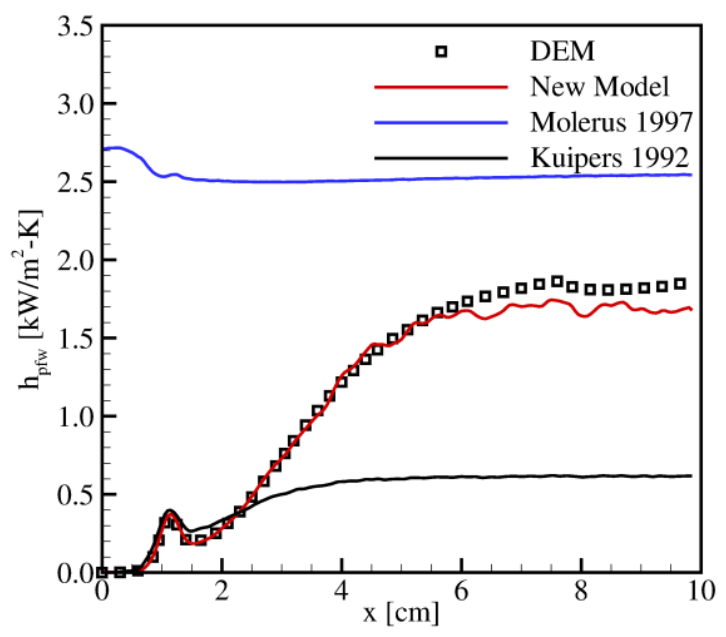

Figure 8. The particle-fluid-wall heat transfer coefficient along the surface of an inclined ramp. The new model and existing models in the literature $[13,22]$ are compared to DEM data.

The DEM data show a small peak in the heat transfer at a distance near $x=1 \mathrm{~cm}$. This peak occurs because several particles strike the ramp and scatter up the slope, forming a small stagnation region with higher solids concentration and increased heat transfer. The new model is able to reproduce this small peak in the local heat transfer coefficient and also closely agrees with DEM data over the entire length of the ramp. After a distance approximately $6 \mathrm{~cm}$ down the ramp, the solids concentration reaches a maximum value and the heat transfer coefficient becomes nearly constant. The new model predicts a slightly smaller value for the maximum heat transfer by approximately $8 \%$. The Nusselt number correlation by Molerus [22] is derived for packed beds and thus does not depend on solids concentration. Consequently, this treatment results in significant over-prediction of the heat transfer coefficient near the top of the ramp where the flow is still relatively dilute. Near the end of the ramp, where the solids concentration and local heat transfer coefficient asymptote, Molerus's correlation for packed beds over predicts the heat transfer by approximately $35 \%$, again tracing to the packed bed limit assumed in their theory. The thermal conductivity model by Kuipers uses Fourier's law to compute the local heat flux according to Eq. 1. Kuipers's model is for heat penetration into the bed (not contact heat transfer) and the effective thermal conductivity depends on the solids concentration and an assumed contact area fraction, but does not depend on the particle diameter. The thermal conductivity model closely predicts the local heat transfer coefficient near the top of the ramp where the flow is dilute but under-predicts the maximum heat transfer by a factor of $300 \%$.

To better characterize the accuracy of the new model, ramp simulations are performed for different parameters and the DEM data are compared to the new model predictions. Figure 9 shows 200, 300, and $500 \mu \mathrm{m}$ particles flowing down a ramp inclined at an angle of $45^{\circ}$. The particle-particle and particle-wall coefficients of restitution were 0.9 , the minimum conduction distance, $s$, was $2.75 \times 10^{-6} \mathrm{~cm}$ and the lens radius is $40 \%$ larger than the particle radius. 


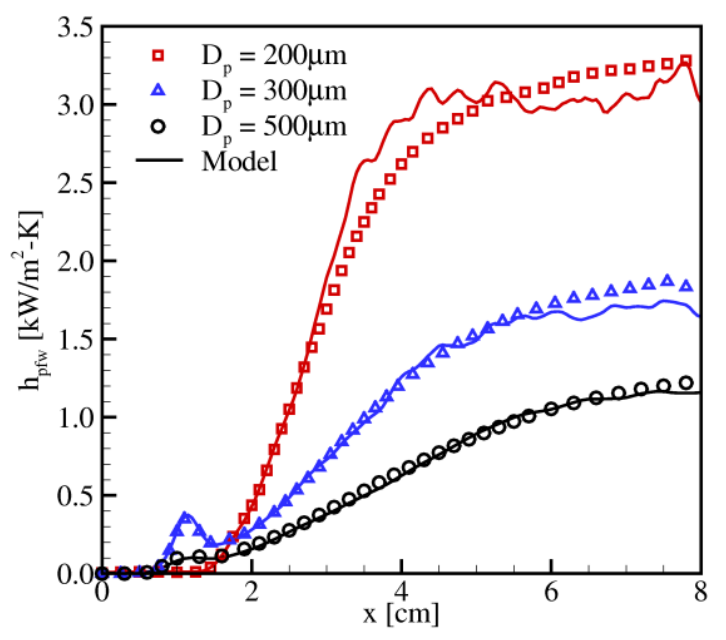

Figure 9. Comparisons of the new model (lines) to DEM data (symbols) for particle diameters ranging from 200 to $500 \mu \mathrm{m}$

The heat transfer coefficient increases as particle size decreases, a trend commonly seen in experiments [18-20]. This increase occurs because there are more small particles per unit wall area than large particles. In addition, the average gap distances, $l$ in Eq. 3, between the wall and a particle decrease for smaller particles, thus reducing the thermal contact resistance and improving the heat transfer. Such particle size effects are not captured by effective solids phase thermal conductivity models that use Fourier's law [13] because in those derivations the thermal conductivity is a continuum property and does not depend on particle diameter. The new model accurately captures particle size effects and predicts a local heat transfer coefficient in good agreement with DEM data over the entire ramp surface. The new model shows larger fluctuations as the heat transfer coefficient asymptotes to a maximum value and the particles closely pack. This occurs because the normalized Nusselt number is most sensitive to changes in concentration near the packing limit, a trend previously shown in Figure 7.

In the next set of simulations, the inclination angle of the ramp is varied and the model predictions are compared to DEM simulations. Figure 10 shows $300 \mu \mathrm{m}$ particles flowing down ramps inclined $30^{\circ}$ to $60^{\circ}$. 


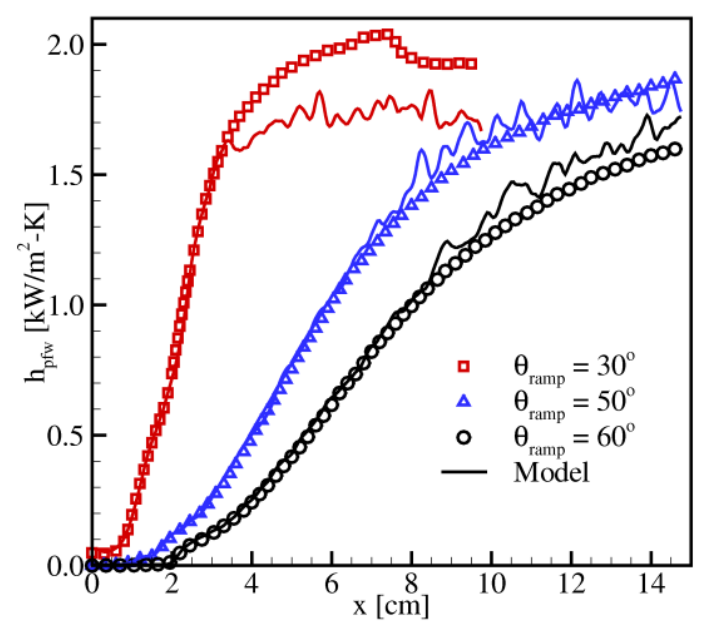

Figure 10. Comparisons of the new model (lines) to DEM data (symbols) for ramp angles ranging from $30^{\circ}$ to $60^{\circ}$

The new model accurately predicts the local heat transfer coefficient for steep ramp angles of $50^{\circ}$ and $60^{\circ}$, but noticeably under-predicts the maximum heat transfer coefficient for a shallower $30^{\circ}$ ramp. Such a discrepancy may be attributed to either the areal number density of particles or the particle-wall distribution function having a dependence on ramp angle. In Figure 11 the number density (per area) of particles within a lens thickness of the wall are plotted versus the solids concentration for multiple ramp angles. Those results show that the particle areal density is independent of ramp angles between $30^{\circ}$ and $60^{\circ}$. As a result, the under-prediction of the heat transfer coefficient for a $30^{\circ}$ ramp likely occurs because the particle-wall distribution function is not solely dependent on the solids concentration but also depends on the inclination angle of the ramp. Physically, a higher percentage of particles are in contact with the wall for shallow inclination angles. This increases the heat transfer coefficient by skewing the particlewall distribution function towards more particles in contact. 


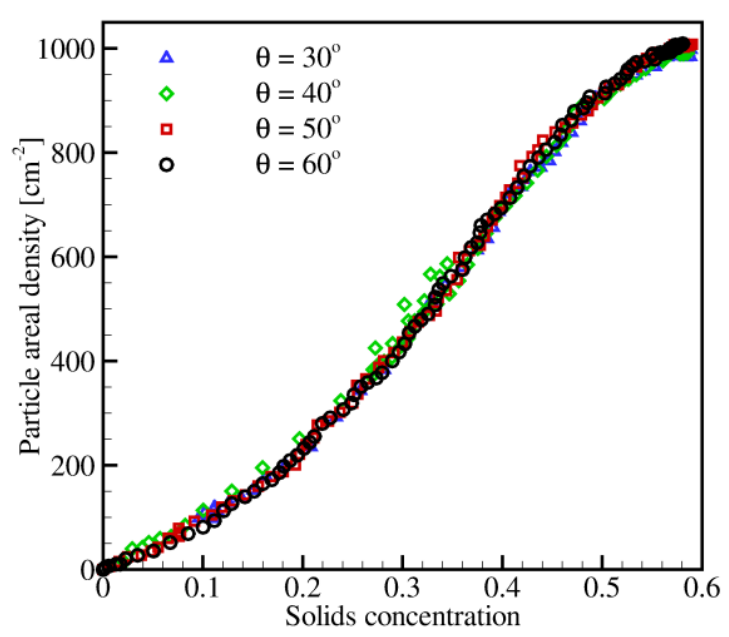

Figure 11. Number of $300 \mu \mathrm{m}$ particles per unit area plotted versus solids concentration for different ramp angles.

The coefficient of restitution has a noticeable impact on the heat transfer. Figure 12 shows $300 \mu \mathrm{m}$ particles flowing down a ramp where the particle-wall and particleparticle restitution coefficients change from a nominal value of 0.9 to a more dissipative value of 0.7. The maximum heat transfer coefficient increases as the restitution coefficient decreases. This occurs because in a more dissipative system the granular temperature decreases and a larger fraction of particles contact the surface. Although the new model is accurate in moderately dense and dilute regions (i.e. $x<2.5 \mathrm{~cm}$ for $e=0.7$ and $x<3 \mathrm{~cm}$ for $e=0.8$ ), it does not predict the dependence of maximum heat transfer to the restitution coefficient in the dense regions. 


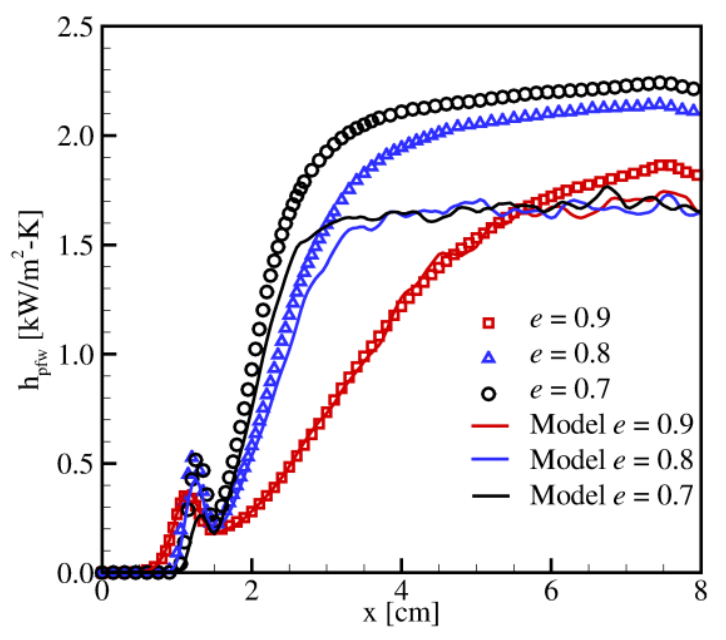

Figure 12. Local heat transfer coefficient comparisons of the new model (lines) to DEM data (symbols) for coefficients of restitution ranging from 0.7 to 0.9

In discrete element simulations, it is common to artificially soften the spring constant to increase the computational efficiency. By softening the spring constant, the collision time increases and therefore allows use of a larger computational time step. This softening procedure can lead to unrealistically long collision durations and large contact areas. Although these effects can drastically increase direct conductive heat transfer across the mutual contact area, the spring constant has less of an impact on the indirect conduction modeled in this work. Figure 13 shows simulations of $300 \mu \mathrm{m}$ particles flowing down a ramp where one has a LSD spring constant of $100 \mathrm{~N} / \mathrm{m}$ and the other has a value of $500 \mathrm{~N} / \mathrm{m}$. The softer spring constant predicts a slightly larger heat transfer coefficient by approximately 7\%. This increase occurs because the contact duration increases as the spring constant softens and particles are more likely to spend more time in contact with the wall. A softer spring constant can also increase the deformation of the particle. The thermal conductance through the interstitial gas for a single particle in contact with a wall is not sensitive to the degree of overlap. Although the overlap reduces the average distance between the particle surface and the wall, the area over which conduction through the interstitial gas occurs decreases. These effects offset and result in the conductance not being sensitive to overlap. 


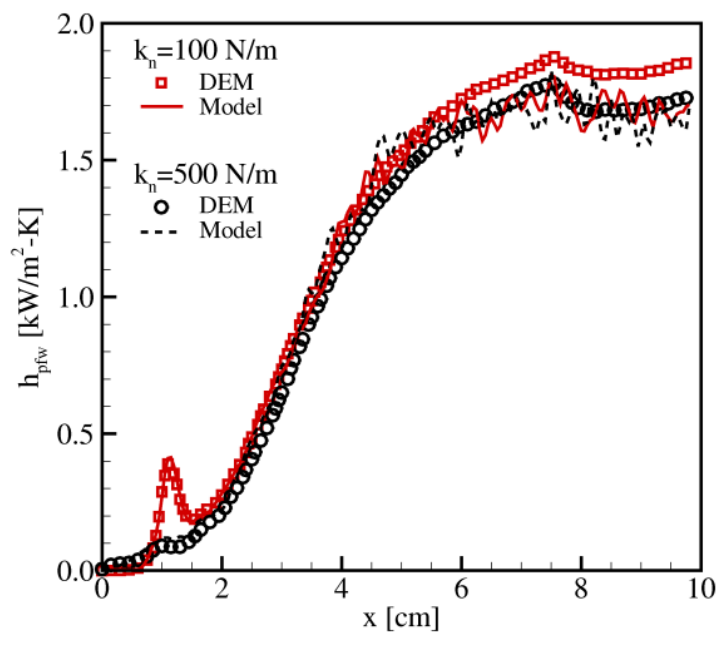

Figure 13. Local heat transfer coefficient comparisons for the new model and DEM simulations with different LSD spring constants

\subsubsection{Full Continuum model (no DEM inputs)}

By using DEM data for the solids concentration etc. as inputs for the continuum model, the previous section isolated the impact of the new model for heat transfer at the boundary on the predictions. In practice, however, two-fluid model simulations yield hydrodynamic variables that may be different from DEM simulations; the corresponding ramifications on the heat transfer predictions are discussed below.

Two-fluid model simulations were performed for 200 and $300 \mu \mathrm{m}$ particles flowing down a ramp with an inclination angle of $45^{\circ}$. The resulting continuum heat transfer predictions are compared to DEM data. The Nusselt numbers, defined in Eq. 8, are plotted along the surface of the ramp in Figure 14 for 200 and $300 \mu \mathrm{m}$ particles. The simulations show excellent agreement for the maximum Nusselt numbers as well as good agreement near the top of the ramp where the flow is relatively dilute, i.e. $\varepsilon_{s}<0.5$. Noticeable discrepancies in the predicted heat flux appear as the solids phase transitions from dilute to dense regimes $(x \sim 2 \mathrm{~cm})$. This occurs because the two-fluid model overpredicts the solids concentration in this region. Although the discrepancies in solids concentration are relatively small, these differences are amplified for heat transfer. Recall from Figure 7 that the Nusselt number correlations in the new model are very sensitive to the solids concentration when near the packing limit. For example, the maximum error for $200 \mu \mathrm{m}$ particles shows the two-fluid model predicting twice as much heat transfer as DEM data, while the error in solids concentration is only $8 \%$. The previous results, however, show that the model is very accurate when accurate values for the solids concentration are used as inputs. Therefore, significant improvement of the heat transfer is expected by refining the two-fluid simulations by using more accurate constitutive relations for the dense granular flow regimes. 


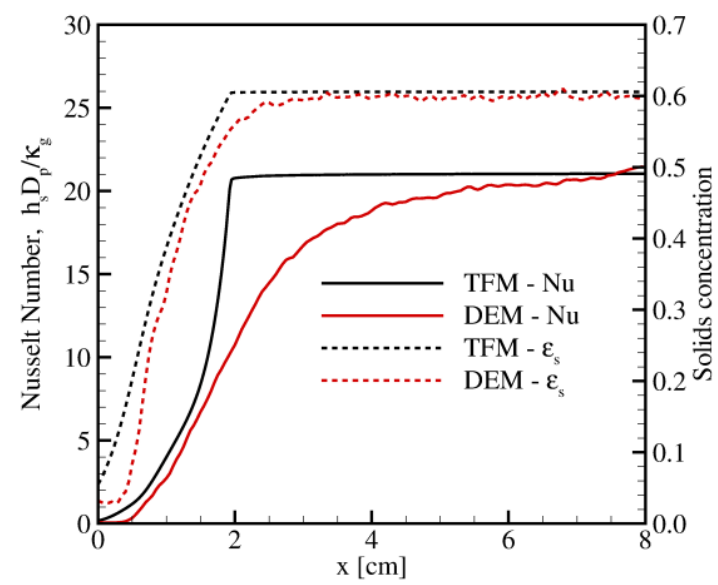

(a)

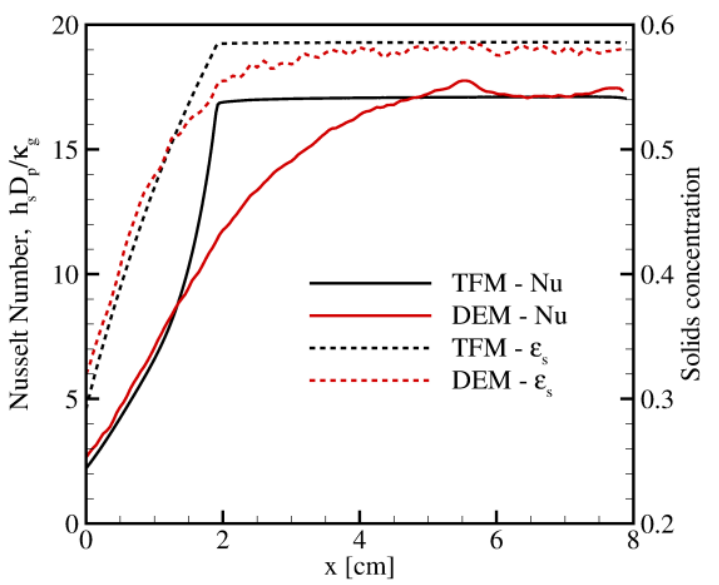

(b)

Figure 14. Nusselt number (solid lines) and solids concentration (dashed lines) comparisons between TFM and DEM simulations. (a) $200 \mu \mathrm{m}$ and (b) $300 \mu \mathrm{m}$ particles

\subsection{Flow over a hexagon}

In this simulation, particles flow around a $4 \times 4$ array of hexagonal tubes, and the heat transfer for a single hexagon within the array is analyzed. The particle diameter was $300 \mu \mathrm{m}$ and the coefficient of restitution was 0.9. Particles enter the domain from the top boundary and then flow through the hexagonal array via gravity. The inlet mass flux and solids concentrations were $258 \mathrm{~kg} / \mathrm{m}^{2}$-s and $10 \%$, respectively. A gas velocity of $10 \mathrm{~cm} / \mathrm{s}$ was prescribed at the inlet and a pressure outflow is used along the bottom domain boundary. The hexagons were $3.78 \mathrm{~cm}$ wide and $6.42 \mathrm{~cm}$ tall and the apex angle, or internal angle measured at the top of the hexagon, was $89^{\circ}$. The geometry is constructed using the Cartesian cut-cell implementation in MFIX [40]. Cut-cells can be used to represent complex geometries. The computational cells along a boundary are truncated to conform to the specified geometry. These results focus on a single hexagon in the $2^{\text {nd }}$ row where a dense stream of particles impinges on its apex. The particles flow down the top surface and then separate at the edges formed along the side walls. The solids concentration field is obtained from DEM data and shown in Figure 15a. The DEM hydrodynamic variables are used as inputs for the new continuum heat transfer model and the local heat transfer coefficients for the new model and DEM data are shown along the top surface in Figure 15b. 

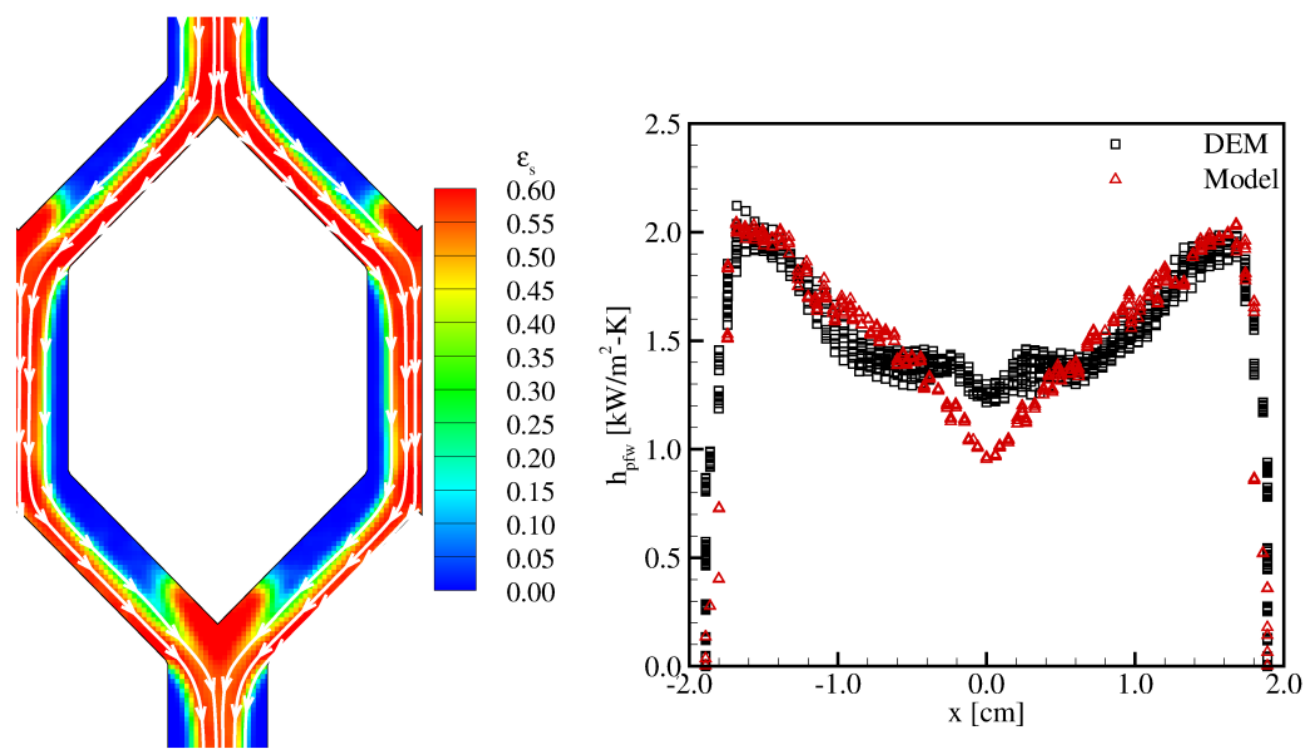

Figure 15. (a) Solids concentration and particle streamlines for a dense flow impinging on a hexagon. (b) Local heat transfer coefficient comparisons along the top surface of the hexagon between the new model and 3-D DEM data.

The new model accurately predicts the local heat transfer coefficient along the top surface of the hexagon. Near the apex the solids concentration has a slightly lower value because the stresses at the impingement point increases the granular temperature. Consequently, the predicted local heat transfer coefficient near the apex is slightly underpredicted, but the model accuracy improves as the flow down the inclined surface develops. The integrated heat transfer coefficient over the entire hexagon has an error less than $4 \%$.

\subsection{Flow over a cylinder}

Particle crossflow around a cylinder is examined to test the robustness of the model for a geometry with curvature. In this simulation, $300 \mu \mathrm{m}$ particles enter the computational domain with a specified mass flux and solids concentration of $258 \mathrm{~kg} / \mathrm{m}^{2}-\mathrm{s}$ and $10 \%$ respectively. The particle-wall coefficient of restitution was 0.9 . The cylinder diameter was $3 \mathrm{~cm}$ and was placed $3.5 \mathrm{~cm}$ below the top inflow plane. The particles impinge on a heated cylinder and form a dense layer of particles along the top surface. This dense particle layer then separates from the surface of the cylinder at a polar angle of approximately $60^{\circ}$ measured from the stagnation point. DEM data for hydrodynamic variables are used as inputs for the new model. The resulting local heat transfer coefficients as predicted by the new model and DEM data are shown in Figure 16. The new model closely matches the heat transfer measured from DEM simulations. Although agreement is excellent near the stagnation point, the new model shows a slightly wider profile than DEM data. This discrepancy can occur because of challenges resolving the solids concentration at the surface. The solids concentration is a continuum field that is averaged over cells that are larger than an individual particle. Consequently, the concentration field is smeared and results in high surface concentrations being predicted despite the particles layer separating from the surface. There are oscillations in the model 
that occur near the stagnation point. These oscillations are a result of numerical artifacts associated with sampling solids concentration in cut-cells with very small volumes.

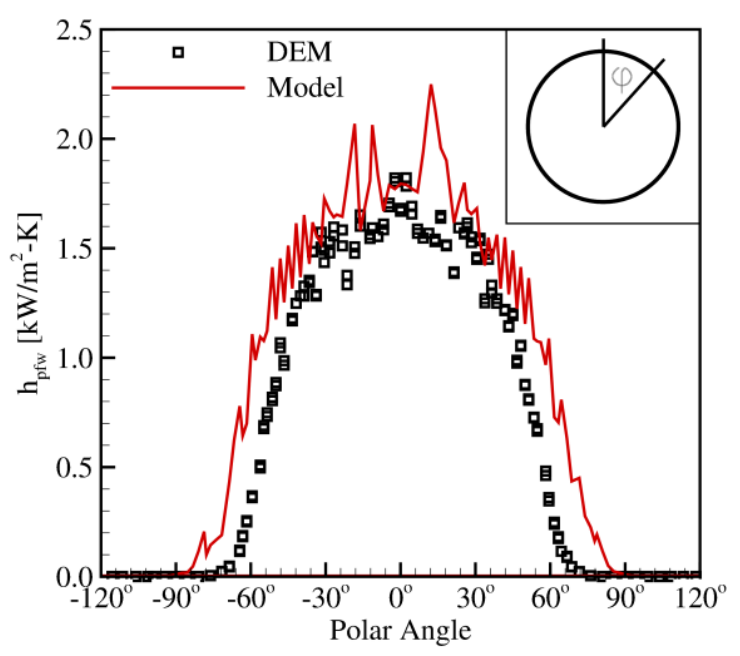

Figure 16. Comparisons of the local heat transfer coefficient between the new model and DEM data for crossflow over a cylinder

\section{Conclusions}

A new fundamental continuum model is developed for solids phase conduction between moving particles and an immersed boundary. This conduction model is for systems where particle-fluid-wall conduction plays a dominant role over direct particlewall conduction. Such systems are common for flowing sands or other materials with similar thermal properties (i.e., $R_{p} \kappa_{g} / R_{c} \kappa_{p}>1$ ). The model is derived by extending the theory for single particle conduction at a wall to a Nusselt number correlation for a continuum of particles near a wall. The heat transfer is very sensitive to the proximity of particles with respect to the wall, i.e. variations in small separation distances from the wall can have a large impact on the heat transfer. To capture such effects, a particle-wall distribution function is incorporated into the model. The particle-wall distribution function is analogous to the more common radial distribution function and describes the probability of a particle existing a specified distance from the wall. Instead of explicitly solving for the particle-wall distribution function, a Nusselt number expression that accounts for the particle-wall distribution function can be obtained by assuming the particle-wall distribution is only dependent on solids concentration. DEM simulations confirm this hypothesis, demonstrating that the Nusselt number data collapse versus solids concentration for a variety of flow conditions. The approximate Nusselt number expression was extracted from DEM data for flow down a ramp, and is used in the new continuum model for a variety of flow conditions and configurations.

The new continuum model for heat transfer at the boundary was tested for uniform particle flow down an inclined ramp, impingement of densely packed particles on the apex of a hexagon, and particle crossflow around a cylinder. The new model 
accurately predicts the local heat transfer coefficient for a range of flow conditions: particle sizes, ramp inclination angles, solids phase mass flow rates, and numerical parameters such as the LSD spring constant. In addition, the model was robust and yielded accurate predictions for the total (or spatially integrated) heat transfer for all geometries investigated in this work. The local heat transfer coefficients for the cylinder case agree well near the stagnation point but larger errors are observed where the particles separate from the surface. This occurred because of challenges resolving particle separation in computational cells that are larger than the particle diameter. The worst comparisons between the new model and DEM data occurred when the restitution coefficient was decreased from 0.9 to 0.7 . At low values of the restitution coefficient the maximum heat transfer was under-predicted by approximately $25 \%$. At higher values, the agreement was significantly better and the model predicted values within $7 \%$ of DEM data. The new conduction model is very sensitive to the solids concentration and accurate predictions for the concentration are critical for model accuracy.

\section{Acknowledgments}

The work was funded by the DOE BRIDGE program as part of the SunShot initiative (grant no. DE-EE0005954). This research used resources of the Oak Ridge Leadership Computing Facility located in the Oak Ridge National Laboratory, which is supported by the Office of Science of the Department of Energy under Contract DEAC05-00OR22725. Simulations were also performed on the Janus supercomputer, which is supported by the National Science Foundation (award number CNS-0821794), the University of Colorado Boulder, the University of Colorado Denver, and the National Center for Atmospheric Research. The Janus supercomputer is operated by the University of Colorado Boulder.

\section{References}

[1] R. Bradshaw, N. Siegel, (2008), "Molten Nitrate Salt Development for Thermal Energy Storage in Parabolic Trough Solar Power Systems," in Proceedings of Energy Sustainability, Jackson, FL.

[2] N.P. Siegel, C.K. Ho, S.S. Khalsa, G.J. Kolb, (2010), "Development and Evaluation of a Prototype Solid Particle Receiver: On-Sun Testing and Model Validation," Journal of Solar Energy Engineering-Transactions of the Asme, 132 (2).

[3] G. Flamant, D. Gauthier, H. Benoit, J.L. Sans, B. Boissiere, R. Ansart, M. Hemati, (2014), "A new heat transfer fluid for concentrating solar systems: Particle flow in tubes," Proceedings of the Solarpaces 2013 International Conference, 49, pp. 617-626.

[4] P.A. Cundall, O.D.L. Strack, (1979), "A discrete numerical model for granular assemblies," Geotechnique, 29, pp. 47-65.

[5] T. Pöschel, T. Schwager, (2005), Computational Granular Dynamics, SpringerVerlag, New York.

[6] Y. Guo, J.S. Curtis, (2015), "Discrete Element Method Simulations for Complex Granular Flows," Annual Review of Fluid Mechanics, 47 (1), pp. 21-46. 
[7] W.L. Vargas, J.J. McCarthy, (2001), "Heat conduction in granular materials," Aiche Journal, 47 (5), pp. 1052-1059.

[8] M. Kwapinska, G. Saage, E. Tsotsas, (2008), "Continuous versus discrete modelling of heat transfer to agitated beds," Powder Technology, 181 (3), pp. 331-342.

[9] B. Chaudhuri, F.J. Muzzio, M.S. Tomassone, (2006), "Modeling of heat transfer in granular flow in rotating vessels," Chemical Engineering Science, 61 (19), pp. 63486360.

[10] W.L. Vargas, J.J. McCarthy, (2002), "Conductivity of granular media with stagnant interstitial fluids via thermal particle dynamics simulation," International Journal of Heat and Mass Transfer, 45 (24), pp. 4847-4856.

[11] J. Wunschmann, E. Schlünder, (1980), "Heat transfer from heated surfaces to spherical packings," International Chemical Engineering, 20 (4), pp. 555-563.

[12] P. Zehner, E. Schlünder, (1970), "Wärmeleitfähigkeit von Schüttungen bei mäßigen Temperaturen," Chemie Ingenieur Technik, 42 (14), pp. 933-941.

[13] J.A.M. Kuipers, W. Prins, W.P.M. Vanswaaij, (1992), "Numerical calculation of wall-to-bed heat transfer coefficients in gas-fluidized beds," Aiche Journal, 38 (7), pp. 1079-1091.

[14] W. Van Antwerpen, C. Du Toit, P. Rousseau, (2010), "A review of correlations to model the packing structure and effective thermal conductivity in packed beds of monosized spherical particles," Nuclear Engineering and design, 240 (7), pp. 1803-1818.

[15] E. Tsotsas, (2010), D6.3 Thermal conductivity of packed beds. In Heat Atlas, $2^{\text {nd }}$ Edition. VDI Gesellschaft (Ed.), Springer, Dusseldorf, pp. 570-613.

[16] E. Tsotsas, (2010), M6 Heat transfer from a wall to stagnant and mechanically agitated beds. In Heat Atlas, $2^{\text {nd }}$ Edition. VDI Gesellschaft (Ed.), Springer, Dusseldorf, pp. 1311-1326.

[17] M. Syamlal, D. Gidaspow, (1985), "Hydrodynamics of fluidization - prediction of wall to bed heat-transfer coefficients," Aiche Journal, 31 (1), pp. 127-135.

[18] A. Denloye, J. Botterill, (1977), "Heat transfer in flowing packed beds," Chemical Engineering Science, 32 (5), pp. 461-465.

[19] M. Hyde, H. Klocke, (1980), "Heat transfer between fluidized beds and heat exchange installations," International Chemical Engineering, 20 (4), pp. 583-599.

[20] M. Golob, S. Jeter, D. Sadowski, (2010), "Heat transfer from flat surfaces to moving sand," in ASME early career technical conference, Atlanta, Georgia.

[21] J.K. Spelt, C.E. Brennen, R.H. Sabersky, (1982), "Heat transfer to flowing granular material," International Journal of Heat and Mass Transfer, 25 (6), pp. 791-796.

[22] O. Molerus, (1997), "Heat transfer in moving beds with a stagnant interstitial gas," International Journal of Heat and Mass Transfer, 40 (17), pp. 4151-4159.

[23] M. Syamlal, W. Rogers, T.J. O’Brien, (1993), "MFIX documentation: Theory guide," Technical Note, DOE/METC-95/1013 and NTIS/DE95000031, U.S. Department of Energy, Morgantown, WV.

[24] S. Pannala, M. Syamlal, T. O'Brien, (2010), Computational Gas-Solids Flows and Reacting Systems: Theory, Methods and Practice: Theory, Methods and Practice, IGI Global, Hershey.

[25] D. Gidaspow, (1994), Multiphase flow and fluidization: continuum and kinetic theory descriptions, Academic Press, San Diego. 
[26] R. Jackson, (2000), The dynamics of fluidized particles, Cambridge University Press, New York.

[27] V. Garzó, J. Dufty, (1999), "Dense fluid transport for inelastic hard spheres," Physical Review E, 59 (5), pp. 5895.

[28] J. Ding, D. Gidaspow, (1990), "A bubbling fluidization model using kinetic theory of granular flow," AIChE Journal, 36 (4), pp. 523-538.

[29] D. Gunn, (1978), "Transfer of heat or mass to particles in fixed and fluidised beds," International Journal of Heat and Mass Transfer, 21 (4), pp. 467-476.

[30] G. Batchelor, R. O'Brien, (1977), "Thermal or electrical conduction through a granular material," Proceedings of the Royal Society of London. A. Mathematical and Physical Sciences, 355 (1682), pp. 313-333.

[31] D. Rong, M. Horio, (1999), "DEM simulation of char combustion in a fluidized bed," in Second International Conference on CFD in the Minerals and Process Industries, Melbourne, Australia, pp. 65-70.

[32] C. Wen, T. Chang, (1967), "Particle to particle heat transfer in air fluidized beds," in Proceedings of International Symposium on Fluidization, Eindhoven, pp. 491-506.

[33] C. Delvosalle, J. Vanderschuren, (1985), "Gas-to-particle and particle-to-particle heat transfer in fluidized beds of large particles," Chemical Engineering Science, 40 (5), pp. 769-779.

[34] G. Cheng, A. Yu, P. Zulli, (1999), "Evaluation of effective thermal conductivity from the structure of a packed bed," Chemical Engineering Science, 54 (19), pp. 41994209.

[35] A. Xavier, J. Davidson, (1978), "Heat transfer to surfaces immersed in fluidized beds, particularly tube arrays", in: Fluidization, Cambridge University Press, Cambridge, pp. 333-338.

[36] W.L. Vargas, J. McCarthy, (2002), "Conductivity of granular media with stagnant interstitial fluids via thermal particle dynamics simulation," International Journal of Heat and Mass Transfer, 45 (24), pp. 4847-4856.

[37] J.L. Lebowitz, (1964), "Exact solution of generalized Percus-Yevick equation for a mixture of hard spheres," Phys. Rev., 133 (4A), pp. A895-A899.

[38] H. Al-Ansary, A. El-Leathy, Z. Al-Suhaibani, S. Jeter, D. Sadowski, A. Alrished, M. Golob, (2012), "Experimental study of a sand-air heat exchanger for use with a hightemperature solar gas turbine system," Journal of Solar Energy Engineering, 134 (4), pp. 041017.

[39] P.C. Johnson, R. Jackson, (1987), "Frictional-collisional constitutive relations for granular materials, with application to plane shearing," Journal of Fluid Mechanics, 176, pp. 67-93.

[40] J.F. Dietiker, T.W. Li, R. Garg, M. Shahnam, (2013), "Cartesian grid simulations of gas-solids flow systems with complex geometry," Powder Technol., 235, pp. 696-705. 